WM-95-105

\title{
Electroproduction and Hadroproduction of Light Gluinos
}

\author{
C. E. Carlson, G. D. Dorata, D. Morgan, and M. Sher \\ Physics Department, College of William and Mary, Williamsburg, VA 23187, USA
}

\begin{abstract}
In a class of supergravity models, the gluino and photino are massless at tree level and receive small masses through radiative corrections. In such models, one expects a gluino-gluon bound state, the $R_{0}$, to have a mass of between 1.0 and $2.2 \mathrm{GeV}$ and a lifetime between $10^{-10}$ and $10^{-6}$ seconds. Applying perturbative QCD methods (whose validity we discuss), we calculate the production cross sections of $R_{0}$ 's in $e-p, \pi-p, K-p, \bar{p}-p$ and $p-p$ collisions. Signatures are also discussed.
\end{abstract}




\section{INTRODUCTION}

In searches for supersymmetric particles, it is generally assumed that the masses of the new particles are $O(100-1000) \mathrm{GeV}$, and thus they can only be produced in high energy accelerators. However, a possibility which has been receiving increasing attention of late 11 9 is that the gluino and photino are extremely light, with masses in range of hundreds of $\mathrm{MeV}$. If so, then the gluino-gluon bound state, called the glueballino (which we designate as $R_{0}$ ), would have a mass in the $1-2 \mathrm{GeV}$ range, and would be very long-lived, possibly with a lifetime as long as that of the muon. The possibility that a strongly interacting, long-lived particle with a mass only slightly greater than that of the neutron could have evaded detection is astonishing, and yet this appears to be the case: An $R_{0}$ mass between 1.0 and $2.2 \mathrm{GeV}$ would not yet have been experimentally excluded [3].

Why would one expect gluinos to be so light? The fact that scalar quark masses must be greater than the $W$ mass shows that supersymmetry is broken at the scale of at least $O(100)$ $\mathrm{GeV}$. However, the source of gaugino masses in many supergravity models is completely different from the source of scalar masses, since the former arise from dimension-3 SUSYbreaking operators. In some such models, such as those in which SUSY is broken in the hidden sector and there are no gauge singlets [1 6], the dimension-3 SUSY-breaking terms are either absent or suppressed by a factor of the Planck mass. Thus, in these models, the gluino and photinof are massless at tree level. Masses will be generated by radiative corrections; these were calculated by Farrar and Masiero [7], who found that as the typical SUSY breaking scale varies from 100 to $400 \mathrm{GeV}$, the gluino mass decreases from 700 to $100 \mathrm{MeV}$, as the photino mass increases from approximately 400 to $900 \mathrm{MeV}$. Although the photino might, in these models, be somewhat heavier than the gluino, the lightest colorsinglet containing the gluino, the $R_{0}$, will be heavier than the photino, for the same reason that a glueball, comprised of massless gluons, has a mass in the $1-2 \mathrm{GeV}$ range. In fact, if the gluino is light, then the $R_{0}$ mass should be very similar to that expected for the lightest $0^{++}$glueball, i.e. $1.4 \pm .4 \mathrm{GeV}$.

If this is the case, then the photino will then be stable, and an ideal candidate for the dark matter. In fact, Farrar and Kolb [8] have shown that if the ratio of the $R_{0}$ mass to the photino mass is in the range from 1.2 to 2.2, then the relic abundance of photinos is just right to account for the dark matter; this mass range overlaps nicely with the range of masses calculated from radiative corrections. Since the gluino will decay through virtual scalar quark processes, the $R_{0}$ lifetime should be quite long; estimates range from $10^{-10}$ to $10^{-6}$ seconds.

How could such a light, long-lived, strongly interacting particle have escaped detection? [5.9] Missing energy searches (the classic signatures of supersymmetry) require large transverse missing energy, and gluinos would not have been detected if the lifetime is greater than $10^{-10}$ seconds. Beam dump experiments which look for the subsequent interaction of the photino would not be sensitive since the photino cross section is significantly smaller (by a factor of $\left.O\left(m_{W} / m_{s q}\right)^{4}\right)$. Experiments at CUSB [10] and ARGUS [1] look for radiative $\Upsilon$

\footnotetext{
${ }^{1}$ When we say photino in this paper, we are actually referring to the lightest neutralino. However, in models in which the lightest neutralino is extremely light, it tends to be a pure photino state.
} 
decays; these experiments can rule out a region of gluino masses which correspond to $R_{0}$ masses from roughly 2 to $4 \mathrm{GeV}$, for any lifetime; other experiments modify the bounds slightly. These experiments are all discussed by Farrar [5,9], who provides a plot of the region of the mass-lifetime plane excluded by each of these experiments; the region from 1.0 to $1.5 \mathrm{GeV}$ is not excluded for any lifetime, and the region from 1.5 to $2.2 \mathrm{GeV}$ is only excluded for lifetimes between $10^{-6}$ and $10^{-8}$ seconds.

There was some excitement recently [2] about the possibility that the presence of light gluinos could alter the running of the QCD coupling constant between $Q^{2}=m_{\tau}^{2}$ and $Q^{2}=$ $m_{Z}^{2}$. It appears that the value of the QCD coupling at the smaller scale is too high, given its value of the larger scale, and modifying the beta function by inclusion of light gluinos could account for the discrepancy. However, it has been pointed out [2] that the uncertainties in this analysis are large, and that the data, at present, can not be used to either establish or rule out light gluinos. Similar arguments apply to jet production at Fermilab and LEP; the uncertainties are too large. In addition, an additional state at $1.4 \mathrm{GeV}$ has been seen, which could be a gluino-gluino bound state, but distinguishing such a state from other possible exotics, such as hybrids, will not be easy.

In order for experimenters to probe the allowed mass and lifetime range, it is necessary to have reasonably accurate values for the production cross section of gluinos. This is not always easy. For example, Farrar [9] has proposed dearching for $R_{0}$ decays into $\eta+\tilde{\gamma}$ by looking for $\eta$ 's in high-intensity kaon beams. This could certainly establish the existence of gluinos, but the production of $R_{0}$ 's relative to kaons cannot be calculated in perturbative QCD, due to the fact that neutral kaon beams are produced at low transverse momentum. On the other hand, one can compute gluino production cross sections at high $p_{T}$ reliably. The cross section for photoproduction of gluino pairs was calculated [12 recently, with the hope of using the photon tagger in the Large Acceptance Spectrometer at CEBAF. Although this calculation did not directly impose a $\left(p_{T}\right)_{\min }$ cut, such a cut would be done by the experimeters, and the event rates were high enough that this cut would not lower the signal too much 2 . As pointed out there, the long $R_{0}$ lifetime and relatively light mass indicates that high-luminosity, lower energy accelerators will be better suited for exploring the allowed range.

In this paper, we will calculate the electroproduction and hadroproduction cross sections for light gluino pairs. Our primary motivation is as follows. Searching for gluinos in high intensity kaon beams, as suggested by Farrar, may very well be the best way to discover gluinos if they are there. However, the absence of a reliably calculable production cross section will make it difficult for experimenters to exclude regions of the mass-lifetime plane; only regions of the mass-lifetime-production cross section volume can be excluded. In electroproduction and hadroproduction, one can reliably calculate the cross-sections in some kinematic regions, and although such experiments may not be the best way to find gluinos, they do offer the possibility of reliably excluding certain regions of the mass-lifetime plane (given the uncertainties associated with pQCD, of course, which we discuss in the next

\footnotetext{
${ }^{2}$ The signature for $R_{0}$ production in that experiment assumed very light or massless photinos, however, and the mass range expected from the above would likely require a different signature. Signatures of $R_{0}$ production will be discussed later.
} 
section).

We will begin by considering electroproduction, discussing the validity of perturbative QCD as well, and then turn to hadroproduction, calculating cross sections for $\pi p, K p, \bar{p} p$ and $p p$ collisions. We will then discuss experimental signatures of light gluinos.

\section{ELECTROPRODUCTION OF LIGHT GLUINOS}

\section{A. Cross section}

The relevant diagrams for electroproduction are shown in Fig 1., and the square of the resulting matrix element is given in the Appendix. In integrating over phase space, the same procedure was used as in the photoproduction calculation. The integrations over the gluino momenta are performed in the $\vec{r}=0$ reference frame, and then re-expressed in covariant form. The subsequent integration over the outgoing quark momentum is done in the quarkphoton center of momentum frame. We do not integrate over the outgoing electron, instead we will express our results as a differential cross section of the form $E_{l_{2}} d \sigma / d^{3} l_{2}$.

Once we obtain the subprocess cross section, we must embed the target quark in a proton and integrate over the allowed values of $\hat{s}$. We fold the cross section with the distribution functions of the quark in a proton

$$
\begin{aligned}
E_{l_{2}} \frac{d \sigma}{d^{3} l_{2}} & =\int d x \sum_{q} e_{q}^{2} f_{q}(x) E_{l_{2}} \frac{d \hat{\sigma}(\hat{s})}{d^{3} l_{2}} \\
& =\int d x E_{l_{2}} \frac{d \hat{\sigma}(\hat{s})}{d^{3} l_{2}} F_{2 p}(x) / x
\end{aligned}
$$

where $F_{2 p}$ is the proton electromagnetic structure function. We used up-to-date CTEQ distribution functions (specifically CTEQ1L) for all of our calculations.

Figure 2 shows the differential cross section $E_{l_{2}} d \sigma / d^{3} l_{2}$ plotted vs. the energy of the outgoing electron. The incident electron energy is $12 \mathrm{GeV}$ (corresponding to the maximum energy likely to be reached at CEBAF in the near future) and the polar angle of the outgoing elecron is fixed at $15^{\circ}$. We have assumed that each final state gluino will be bound within a glueballino (a gluon/gluino bound state) and in evaluating our formulas, we have given the gluino an effective mass equal to the glueballino mass. Our results are sensitive to this mass, and we have plotted our results for glueballino masses of 1.0,1.2, and $1.5 \mathrm{GeV}$. The results are not sensitive to the quark mass since there are no collinear singularities for spacelike $q^{2}$. Our calculations assigned the quark an effective mass $x m_{N}$ so that the threshold for the $\gamma+q$ subprocess would be at the same photon energy as for the overall $\gamma+p$ process. Letting the quark be massless would make a negligible difference away from threshold.

\section{B. Applicability of pQCD}

The energies and transverse momenta involved here are not very large and one may worry about the validity of calculations based on perturbative QCD. Already our worries should be assuaged by insensitivity to the quark mass displayed in the photoproduction calculation, 
where even using a quark mass as large as $1 \mathrm{GeV}$ has only a small effect on the size of the calculated cross section.

We may study the reliability of pQCD in more detail by considering how off shell the internal propagators are in these calculations. Far off shell means the internal particles can travel only short distances, and short distances are where pQCD is valid. Two of the three propagators in the two diagrams of the photoproduction version of Fig. 1 are always far off shell. These are the quark propagator in the s-channel diagram and the gluon propagator, which has to supply the energy to produce a massive gluino or even a glueballino pair. The quark propagator in the u-channel diagram, however, can get rather close to singular when the photon and outgoing quark are collinear.

We studied the importance of this near singularity in the photoproduction case. First, we control the singularity as we normally do by inserting a quark mass. Then we add an extra requirement, that $|\hat{u}|$ be greater than some fixed amount to ensure that whatever contributions we keep in our calculation are perturbatively reliable. Here, the "hat" denotes a Mandelstam variable for the $\gamma-q$ subprocess. Requiring $|\hat{u}|>1 \mathrm{GeV}^{2}$ (which, if we include the quark mass, means the propagator is off shell by more than $1 \mathrm{GeV}^{2}$ ) leads to a decrease in cross section of less than five percent for incoming photon energies of $10 \mathrm{GeV}$ and glueballino masses in the $1.0-1.5 \mathrm{GeV}$ range. We conclude that the bulk of our cross section comes from kinematics where all internal propagators are far off shell and hence that the perturbative calculations are good approximations to the correct cross section.

\section{Event rates}

The Hall B Large Acceptance Spectrometer at CEBAF can accept a luminosity of $10^{34}$ $\mathrm{cm}^{-2} \mathrm{sec}^{-1}$. (The luminosity for Hall B is set by what the detector can accept rather than by what the accelerator can produce.) For electroproduction, taking $10^{-3} \mathrm{nb} / \mathrm{GeV}^{2}$ as a typical cross section in Fig. 2, this translates into a typical event rate of 1 per 100 seconds. Similar event rates will be obtained for the proposed ELFE accelerator, if it has a large acceptance detector. Even with a lifetime near the upper end of the expected range, one microsecond, one would have an $R_{0}$ decaying in the detector several times per day. Signatures of these decays will be discussed below.

\section{HADROPRODUCTION OF LIGHT GLUINOS}

We will consider two classes of hadroproduction reactions. The first class involves reactions in which the incident particle contains one or more valence $\bar{u}$ or $\bar{d}$ anti-quarks, including $\pi p, K p$ and $\bar{p} p$. Then we will consider production via $p p$ collisions at the end of the section.

If one of the hadrons contains valence anti-quarks, then the dominant mode of gluino production will be through $q \bar{q}$ annihilation (see Fig. 3). The calculation for this process is straightforward, and the resulting cross section is given by 13

$$
\hat{\sigma}=\frac{16 \pi \alpha_{s}^{2}}{9 \hat{s}}\left(\frac{1+2 M^{2}}{\hat{s}}\right) \sqrt{\frac{1-4 M^{2}}{\hat{s}}}
$$


where $\hat{s}$ is the total energy in the quark-photon center of momentum frame, and once again we will consider $M$ to be the glueballino mass. In order to obtain the total cross section, we fold this subprocess cross section with the hadron distribution functions

$$
\sigma=\iint \hat{\sigma} \sum_{q}\left(q_{a}\left(x_{1}\right) \bar{q}_{b}\left(x_{2}\right)+\bar{q}_{a}\left(x_{1}\right) q_{b}\left(x_{2}\right)\right) d x_{1} d x_{2}
$$

where $x_{1}$ and $x_{2}$ are the momentum fractions of the quark and anti-quark, and $q_{a}(x)$ and $q_{b}(x)$ are the quark (anti-quark) distribution functions for each hadron. For the proton, we once again use the CTEQ1L distribution functions. For mesons we will use

$$
\begin{gathered}
v(x)=.75 x^{-1 / 2}(1-x) \\
s(x)=.12 x^{-1}(1-x)^{5}
\end{gathered}
$$

for the valence and sea quark (or anti-quark) distribution functions, respectively. We will also assume " $S U\left(2 \frac{1}{2}\right)$ " for the strange sea quarks, that is, we assume that there are half as many $s \bar{s}$ pairs in the quark sea of the meson as there are $u \bar{u}$ and $d \bar{d}$ pairs.

The results for $K^{-} p, \pi^{-} p$, and $\bar{p} p$ are shown in figures 4,5 , and 6 respectively. The total cross section is plotted versus the incident beam energy for glueballino masses of 1.0, 1.5, and $2.0 \mathrm{GeV}$.

The cross sections are quite high. For example, for the $18 \mathrm{GeV} \pi^{-}$beam at Brookhaven, one has an event rate of roughly $0.5 /$ microbarn/sec. For a $1.0 \mathrm{GeV} R_{0}$, this gives an event every two seconds (for a microsecond lifetime, an $R_{0}$ will decay within a meter of the interaction region every hour or so). For a $2.0 \mathrm{GeV} R_{0}$, the rate is two orders of magnitude smaller.

The second class of hadroproduction reactions involves cases in which the incident particle does not contain any valence anti-quarks, for example, proton-proton collisions. Although the process $q \bar{q} \rightarrow \tilde{g} \tilde{g}$ will still contribute (due to the presence of sea antiquarks in both particles) it will be suppressed relative to the cases in which there are valence anti-quarks. To the same order, there will be a contribution from gluon fusion [13], $g g \rightarrow \tilde{g} \tilde{g}$. The process $g q \rightarrow \tilde{g} \tilde{g} q$, although higher order in the coupling constant, may be competitive with this process Here, the calculation is necessarily imprecise, since the gluon distribution function we use will be modified by the presence of gluinos in the sea (we are omitting contributions from primordial gluinos. Using a conventional gluon distribution function we have found that the two contributions are similar, and that the resulting curves are the same shape as those in Fig. 6, but are roughly two orders of magnitude smaller.

\section{SIGNATURES}

One general signature of the glueballino is that it can aspects of both a long lived and a short lived particle. It should, like a long lived particle, decay a long distance away from where it was produced. Then if it decays into two or more hadrons, it should have a wide decay width in the sense that the spread of mass visible in the decay should be large. This is a consequence of the varying energy taken away by the almost non-interacting photino if the final state is three or more particles in total. It is important in this case that the decay not be one that could be mimicked by known weakly decaying particles. With this 
in mind, it was proposed to look for the decay of the glueballino into four charged pions plus an unobserved photino; the appearance of four charged pions emerging from a vertex away from the interaction point would be a "gold-plated" signature for glueballinos, and the branching ratio of known mesons in this mass range into four charged pions is not unusually small.

This would be the best signature if the photino was very light. However, the work of Farrar and Masiero, and the cosmological arguments of Farrar and Kolb, suggest that the photino is not particularly light, and thus the decay into four charged pions will, if even kinematically allowed, be suppressed significantly. One could look for three pions and a photino, with the three pions having more invariant mass than the kaon.

The two most interesting two-body decays are into $\pi^{o}+\tilde{\gamma}$ and, if kinematically allowed, $\eta+\tilde{\gamma}$. It is the latter decay that Farrar [9] has proposed looking for in experiments that produce kaon beams since there may be some admixture of $R_{0}$ in the beam; the $\eta$ will subsequently decay into three pions with more invariant mass than the kaon. Due to SU(3) factors, the branching ratio of $\eta+\tilde{\gamma}$ will be, to the extent that the $\eta$ mass does not suppress the rate, $10 \%$ of the $\pi^{o}+\tilde{\gamma}$ ratio. The appearance of a single $\pi^{0}$ a distance from the vertex may be difficult to pick out of the background, and the $\eta$ may thus be easier to find.

One could also look for $\pi^{+} \pi^{-} \tilde{\gamma}$ where the pions have an invariant mass greater than the kaon. Although phase space arguments indicate a branching ratio of $O(10)^{-3}$ [9], such arguments generally underestimate the multi-hadron decay rates of mesons in the $1-2 \mathrm{GeV}$ mass range; for many such mesons the multi-hadron decay will dominate the two-body decay. Thus, the branching ratio for this mode could be sizable.

\section{CONCLUSIONS}

It is remarkable that the existence of a long-lived, strongly interacting particle with a mass just slightly above that of the neutron cannot be experimentally excluded. Given that such a particle is a consequence of a class of supergravity models, a comprehensive search for light gluinos is well-motivated. Although the best method of detecting gluinos might well be to look for their presence in kaon beams, the absence of a reliable production cross section precludes the possibility of definitely ruling out gluinos in a given mass and lifetime region. In this article, we have calculated the rate for electroproduction and hadroproduction of light gluinos, in a kinematic regime in which perturbative QCD should be fairly reliable. The event rates are quite high, and the signatures fairly distinctive. Failure to find gluinos at the predicted rate (or within a factor of a few, given the uncertainties in perturbative QCD at this scale) will definitively rule out light gluinos in a given mass-lifetime region. Their discovery will revolutionize particle physics, and lead to a new generation of "gluino factories".

\section{ACKNOWLEDGMENT}

We thank Glennys Farrar, John Kane, Larry Weinstein and Suh-Urk Chung for many useful discussions. This work was supported by the NSF under Grant No. NSF-PHY9306141. 


\section{REFERENCES}

[1] G.R. Farrar, Phys. Rev. Lett. 531029 (1984); S. Dawson, et al., Phys. Rev. D31 1581, (1985); UA1 Collaboration, Phys. Lett. 198B 261, (1987).

[2] G.R. Farrar, Phys. Lett. 265B 395, (1991); R. Munoz-Tapia and W. Stirling, Phys. Rev. D49 3763, (1994); J. Ellis, D. Nanopoulos and D. Ross, Phys. Lett. 305B 375, (1993); M. Schmelling and R. St. Denis, Phys. Lett. 329B 393, (1994); L. Clavelli, Phys. Rev. D45 3276, (1992); L. Clavelli, et al., Phys. Rev. D47 1973, (1993).

[3] G.R. Farrar, Phys. Rev. D51 3904, (1995) has a very detailed analysis of the current experimental situation and an extensive set of references.

[4] T. Banks, D. Kaplan and A. Nelson, Phys. Rev. D49 779, (1994).

[5] G.R. Farrar, preprint RU-95-17, hep-ph/9504295, Rutgers Univ., April 1995.

[6] G.R. Farrar, preprint RU-95-25, Rutgers Univ., July 1995.

[7] G.R. Farrar and A. Masiero, preprint RU-94-38, hep-ph/9410401, Rutgers Univ. 1994.

[8] G.R. Farrar and E.W. Kolb, preprint RU-95-18, astro-ph/9504081, Rutgers Univ. April 1995.

[9] G.R. Farrar, preprint RU-95-26, Rutgers Univ., July 1995.

[10] P.M. Tuts, et al., Phys. Lett. 186B 233, (1987).

[11] ARGUS Collaboration, Phys. Lett. 167B 360, (1986).

[12] C.E. Carlson and M. Sher, Phys. Rev. Lett. 72 2686, (1994).

[13] See P.R. Harrison and C.H. Llewellyn-Smith, Nucl. Phys. B213 223, (1983) (E. Nucl. Phys. B223 542, (1983)) for the full calculation of the subprocess cross-sections. We take the limit in which the squark mass is much greater than the gluino mass, so diagrams with t-channel squark exchange can be neglected.

\section{APPENDIX}

The diagrams for electroproduction of light gluinos are shown in Fig. 1. In terms of the momenta defined in the diagrams, we define, with $m$ being the mass of the gluino,

$$
\begin{aligned}
\Delta & =\left(k_{1}-k_{2}\right) / 2 \\
\Delta^{2} & =m^{2}-r^{2} / 4 \\
s & =\left(p_{1}+l_{1}\right)^{2} \\
t & =\left(p_{1}-l_{2}\right)^{2} \\
s_{h} & =\left(p_{2}+r\right)^{2} \\
t_{h} & =\left(p_{1}-p_{2}\right)^{2} \\
u_{h} & =\left(p_{1}-r\right)^{2} \\
Q & =\left(l_{1}+l_{2}\right) / 2
\end{aligned}
$$

Then, the square of the matrix element is

$$
\begin{aligned}
\overline{|\mathcal{M}|^{2}}= & -\frac{4 e^{2} e_{q}^{2} g_{s}^{4}}{q^{4} r^{4}}\{ \\
& \frac{32}{u_{h}^{2}}\left[\left(1+\frac{u_{h}}{s_{h}}\right) q^{2}\left(s_{h}-r^{2}\right)+\left(s-t-q^{2}-r^{2}+t_{h}-4 l_{2} \cdot r\right)^{2}+q^{2} t_{h}\right]\left(p_{1} \cdot \Delta\right)^{2}
\end{aligned}
$$




$$
\begin{aligned}
& +\frac{32}{s_{h}^{2}}\left[(s-t)^{2}+q^{2} t_{h}-q^{2}\left(r^{2}-u_{h}\right)\left(1+\frac{s_{h}}{u_{h}}\right)\right]\left(p_{2} \cdot \Delta\right)^{2} \\
& +\frac{64}{s_{h} u_{h}}\left[q^{2}\left(s_{h}+t_{h}+u_{h}-2 r^{2}\right)+(s-t)\left(s-t-q^{2}-r^{2}+t_{h}-4 l_{2} \cdot r\right)\right] p_{1} \cdot \Delta p_{2} \cdot \Delta \\
& -\frac{128}{u_{h}}\left(s-t-q^{2}-r^{2}+t_{h}-4 l_{2} \cdot r\right) p_{1} \cdot \Delta Q \cdot \Delta \\
& -\frac{128}{s_{h}}(s-t) p_{2} \cdot \Delta Q \cdot \Delta \\
& +\frac{128}{s_{h} u_{h}}\left[r^{4}-r^{2}\left(s_{h}+t_{h}+u_{h}\right)+s_{h} u_{h}\right](Q \cdot \Delta)^{2} \\
& -\frac{4}{s_{h}^{2}}\left(2 \Delta^{2}+r^{2}\right)\left[q^{2} s_{h} u_{h}-r^{2} q^{2}\left(s_{h}+t_{h}+u_{h}\right)+r^{4} q^{2}\right. \\
& \left.+s_{h}(s-t)\left(q^{2}+r^{2}-t_{h}+4 l_{2} \cdot r\right)-r^{2}(s-t)^{2}\right] \\
& -\frac{4}{u_{h}^{2}}\left(2 \Delta^{2}+r^{2}\right)\left[q^{2} s_{h} u_{h}-r^{2} q^{2}\left(s_{h}+t_{h}+u_{h}\right)+r^{4} q^{2}\right. \\
& -u_{h}\left(s-t-q^{2}-r^{2}+t_{h}-4 l_{2} \cdot r\right)\left(q^{2}+r^{2}-t_{h}+4 l_{2} \cdot r\right) \\
& \left.-r^{2}\left(s-t-q^{2}-r^{2}+t_{h}-4 l_{2} \cdot r\right)^{2}\right] \\
& -\frac{4}{s_{h} u_{h}}\left[2 \Delta^{2} t_{h}\left(q^{2}+r^{2}-t_{h}+4 l_{2} \cdot r\right)^{2}\right. \\
& +\left(s-t-q^{2}-r^{2}+t_{h}-4 l_{2} \cdot r\right)\left(( q ^ { 2 } + r ^ { 2 } - t _ { h } + 4 l _ { 2 } \cdot r ) \left[2 \Delta^{2}\left(r^{2}-u_{h}\right)\right.\right. \\
& \left.\left.-r^{2} t_{h}\right]-r^{2}(s-t)\left(4 \Delta^{2}-2 t_{h}+r^{2}-u_{h}\right)\right) \\
& +\left(q^{2}+r^{2}-t_{h}+4 l_{2} \cdot r\right)(s-t)\left[2 \Delta^{2}\left(s_{h}-r^{2}\right)+r^{2} t_{h}\right] \\
& +2 r^{2} q^{2} t_{h}\left(s_{h}+t_{h}+u_{h}\right)-r^{2}\left(r^{2}-u_{h}\right)\left(s-t-q^{2}-r^{2}+t_{h}-4 l_{2} \cdot r\right)^{2} \\
& +\left(s_{h}-r^{2}\right)\left[4 \Delta^{2} q^{2}\left(r^{2}-u_{h}\right)+r^{2}(s-t)^{2}\right] \\
& \left.\left.+2 r^{2}\left(6 \Delta^{2}+r^{2}\right) q^{2} t_{h}\right]\right\}
\end{aligned}
$$




\section{FIGURES}

FIG. 1. The Feynman diagrams for electroproduction of gluinos. The dashed lines represent the gluinos.

FIG. 2. The differential cross section for electroproduction of glueballino pairs is plotted vs. the energy of the outgoing electron. The incident electron energy is $12 \mathrm{GeV}$ and the polar angle of the outgoing elecron is fixed at $15^{\circ}$. The solid, dashed, and dotted lines show the results for a glueballino mass of $1.0 \mathrm{GeV}, 1.2 \mathrm{GeV}$, and $1.5 \mathrm{GeV}$ respectively.

FIG. 3. The Feynman diagram for the production of gluinos via $q \bar{q}$ annihilation. The dashed lines represent the gluinos.

FIG. 4. The total cross section for $K^{-} p \rightarrow \tilde{g} \tilde{g}+X$ is plotted vs. the energy of the incident kaon beam . We are assuming that all gluinos end up in $R_{0}$ 's, as expected. The heavy, thin, and dashed lines show the results for a glueballino mass of $1.0 \mathrm{GeV}, 1.5 \mathrm{GeV}$, and $2.0 \mathrm{GeV}$ respectively.

FIG. 5. The total cross section for $\pi^{-} p \rightarrow \tilde{g} \tilde{g}+X$ is plotted vs. the energy of the incident pion beam . The heavy, thin, and dashed lines show the results for a glueballino mass of $1.0 \mathrm{GeV}, 1.5$ $\mathrm{GeV}$, and $2.0 \mathrm{GeV}$ respectively.

FIG. 6. The total cross section for $p \bar{p} \rightarrow \tilde{g} \tilde{g}+X$ is plotted vs. the energy of the incident

anti-proton beam. The heavy, thin, and dashed lines show the results for a glueballino mass of 1.0 $\mathrm{GeV}, 1.5 \mathrm{GeV}$, and $2.0 \mathrm{GeV}$ respectively. 

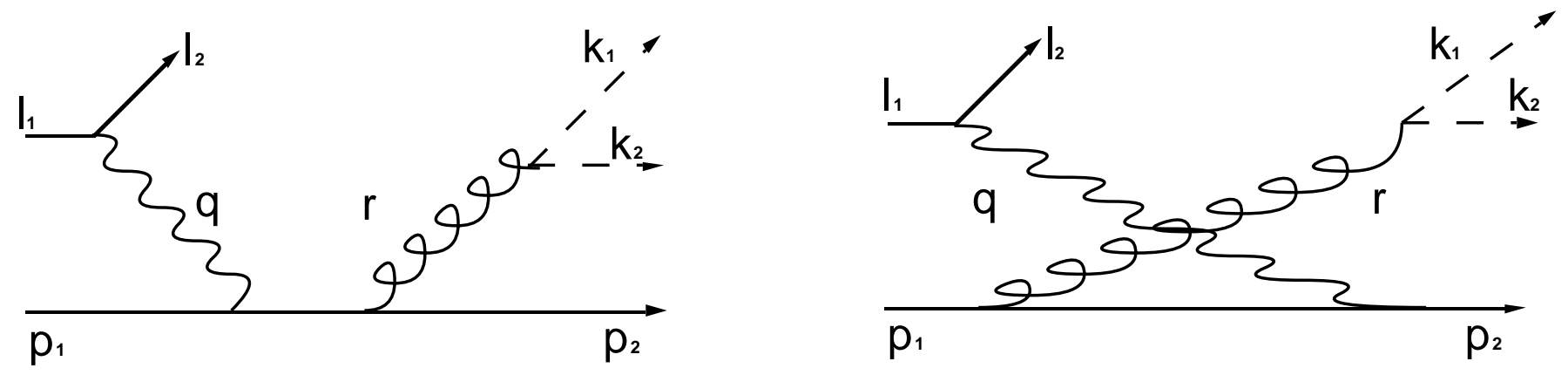

FIG. 1 .

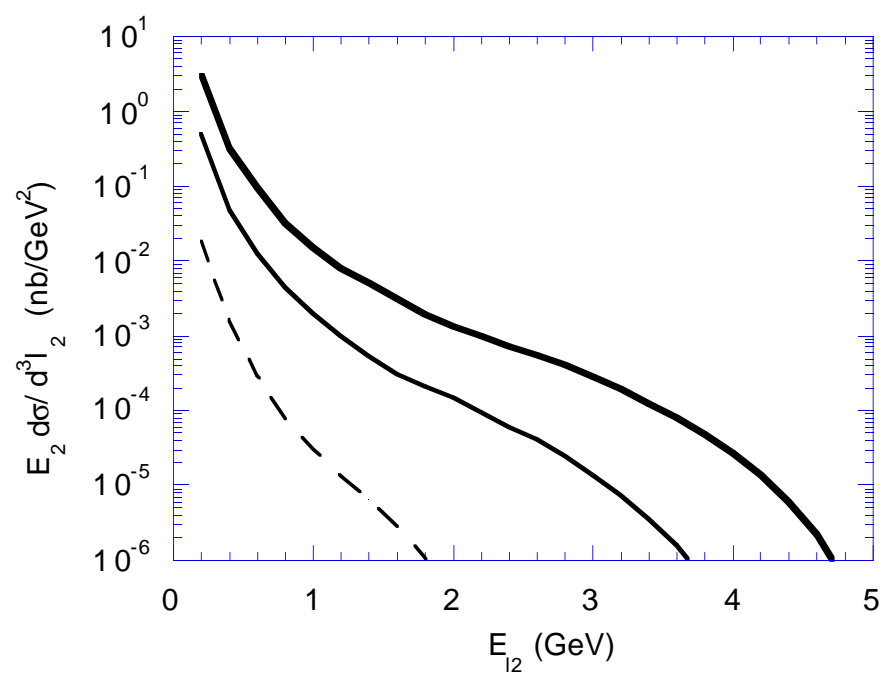

FIG. 2 .

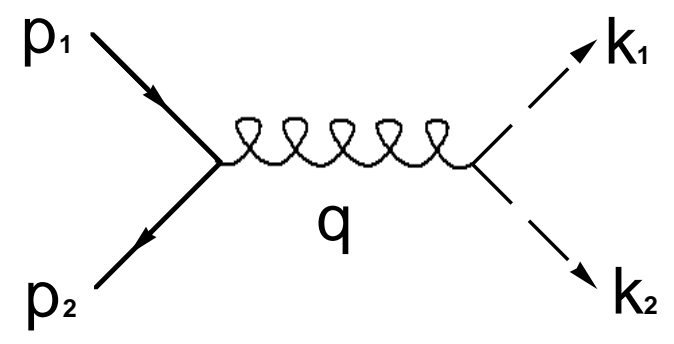

FIG. 3.

1 


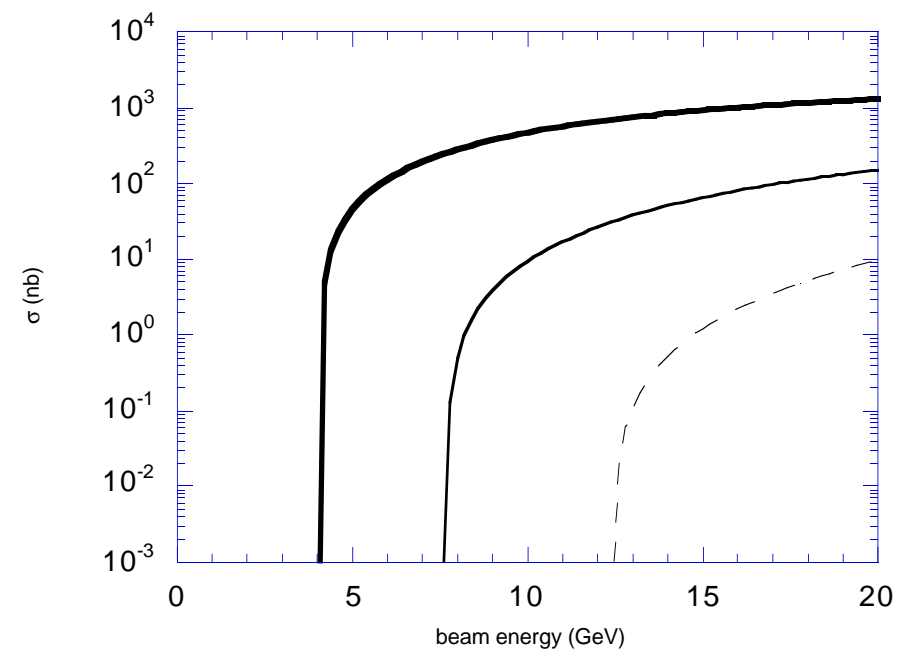

FIG. 4 .

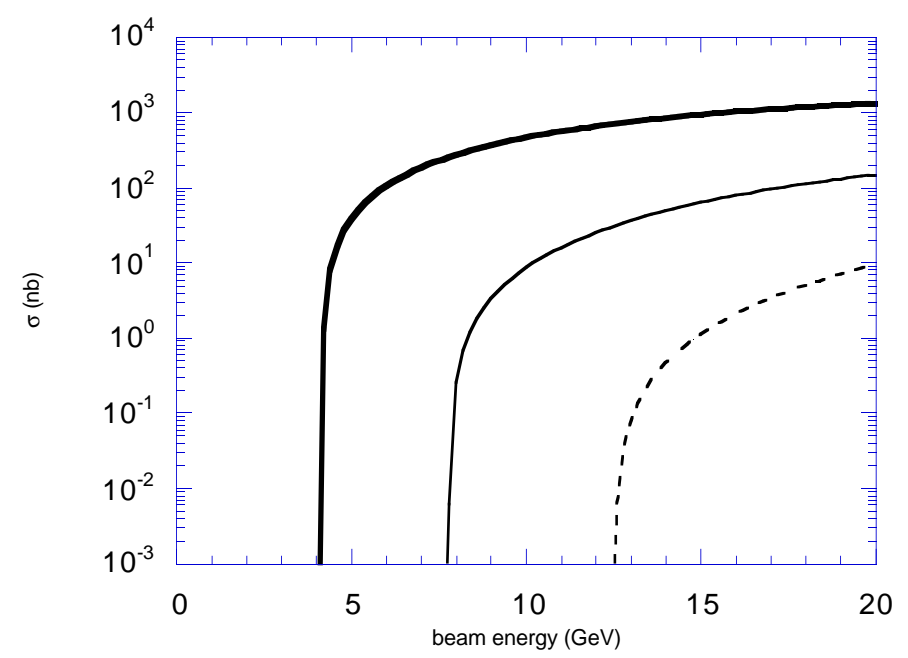

FIG. 5 .

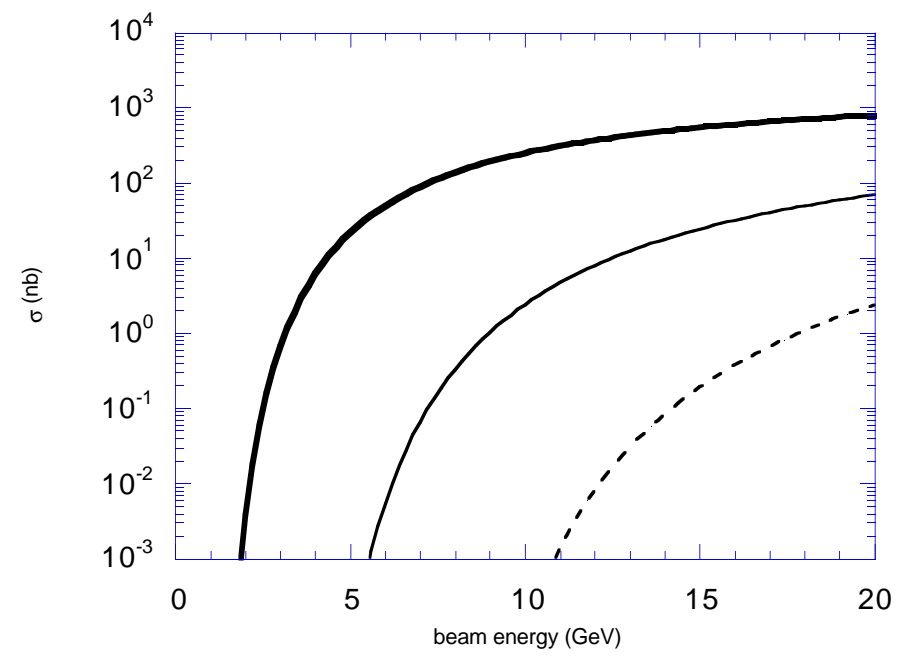


FIG. 6 . 